\title{
Effects of $d$-amphetamine on quantitative measures of motor performance
}

\begin{abstract}
The effects of $10 \mathrm{mg}$. of d-amphetamine and a placebo of similar appearance were determined by means of a battery of quantitative objective measures of motor performance in normal volunteers. The medication was administered in a random, double-blind, crossover design on two occasions one week apart. No significant differences between the effects of the medications on resting and sustention tremor or precision hole steadiness were found. However, several compensatory tracking tasks which required sustained concentration and motor coordination were significantly improved with d-amphetamine.
\end{abstract}

Edward F. Domino, M.D., James W. Albers, Ph.D., Alfred R. Potvin, Ph.D.,* Brian S. Repa, M.S., and Wallace W. Tourtellotte, M.D., Ph.D.** Ann Arbor, Mich. Michigan Neuropsychopharmacology Research Program, The Departments of Pharmacology and Neurology, and the Bioengineering Program, University of Michigan.

It is well known that $d$-amphetamine in proper dosage and circumstance improves human performance on certain behavioral tasks. ${ }^{1,11}$ The effects depend on the dose and mental state of the individual. Oral doses in the order of 10 to $30 \mathrm{mg}$. may reverse behavioral decrements due to fatigue and sleep loss, ${ }^{4}$ increase wakefulness, and increase motor activity. Performance of simple mental tasks may be improved,

Supported in part by United States Public Health Service Grant MH-11846.

Received for publication Sept. 20, 1971.

Accepted for publication Nov. 23, 1971.

- Present address: Department of Electrical Engineering, University of Texas, Arlington, Texas 76010 .

- Present address: Research and Neurology Service, VA Hospital (Wadsworth), Los Angeles, Calif. 90073, and The Department of Neurology, University of California, Los Angeles, Calif. 90024 . although the number of errors is not necessarily decreased. ${ }^{12}$ In general, $d$-amphetamine is known to improve performance in human beings which requires attention despite continued boredom. ${ }^{3,}$ Although many behavioral tasks have been used in studying the effects of $d$-amphetamine in man, there still is a need for precise measures of human motor performance.

This paper describes some effects of a standard dose of $d$-amphetamine given orally on quantitative measures of motor performance in order to stimulate widespread use of such tests in assessing amphetamine-like drugs. It was hypothesized that quantification of motor function would be a very accurate means of determining the effects of small doses of amphetamine in normal nonfatigued subjects. 


\section{Methods}

Six paid male graduate students participated as subjects. A medical history and physical examination were obtained for each subject to ensure normality for the purposes of the study. The subjects were tested on two occasions, one week apart. The oral medications were administered in a random, double-blind, crossover fashion. Each subject received $10 \mathrm{mg}$. of $d$-amphetamine one week and a placebo of similar taste and appearance the other. They were told that they might receive either active or inactive material on one or both occasions. Both experimenters and subjects were unaware of the order of medications; they knew only that an amphetamine-like drug might be given.

Primary interest was to obtain objective measures of the effects of $d$-amphetamine on motor performance. The measures chosen were an extension of the Clinical Quantitative Neurological Examination developed by Tourtellotte and associates. ${ }^{5,7}$, s, 10 The quantitative measures included tests of the following:

1. Tremor-Measures of resting and sustention tremor were obtained with the use of an accelerometer placed on the index finger of the dominant hand. Six 10 second trials were conducted for each test with the average score for the 6 trials used as the test measure. Scores were based on the average of the absolute acceleration. Units are in G-seoonds per second, where $G$ refers to the acceleration due to gravity. For a more complete description of the test apparatus in this and subsequent tests, see Potvin.?

2. Precision hole steadiness-The subject's ability to hold a stylus in a circular hole without touching the sides was used as a measure of steadiness. Both supported and unsupported conditions were used for static and dynamic positioning of the stylus. In the static task, the subject was required to hold the stylus in the smallest possible hole for 5 seconds without contacting the sides of the hole. In the dynamic task, the subject was required to insert the 3 inch stylus $2 \frac{1}{2}$ inches into the hole and then withdraw it without contacting the sides. No time limit was placed on the dynamic task. Six trials were conducted for the 4 tests. The steadiness measure for each of the test conditions was the difference between the diameter of the smallest hole for which the subject made no contact and the diameter of the stylus averaged over 2 trials. Units were in $1 / 128$ of an inch.

3. Tracking-Three compensatory tracking tasks were used to measure the subject's tracking ability. The constant-force tracking task required the subject to apply a constant $300 \mathrm{Gm}$. force to a force stick. A meter was used to display to the subject his deviation from $300 \mathrm{Gm}$. during the test. Six 10 second trials were conducted for both supported and unsupported conditions. The score for a given trial was the average of the subject's absolute deviation from $300 \mathrm{Gm}$. The average score for the six trials, recorded in gram-seconds per second, was used as the test measure.

The random tracking task required the subject to follow a random-appearing signal using a large position control stick. The difference between the subject's output and the desired output was displayed on an oscilloscope screen. Ten 45 second trials were used, the score for each trial being the average of the subject's absolute position error. The average score for the 10 trials, expressed in millimeter-seconds per second was used as the test measure.

The critical tracking task required the subject to control a system which became increasingly unstable with time. The same display and control stick were used as in the random tracking task. The difference between the desired null output of the system and actual output was displayed to the subject. When the subject could no longer keep the control error within the range of the screen, the test was automatically terminated. Scores, given in reciprocal seconds, reflect the highest degree of instability controllable by the subject and are an approximate measure of the reciprocal of the subject's effective time delay. The average of 10 trials was used as the test 


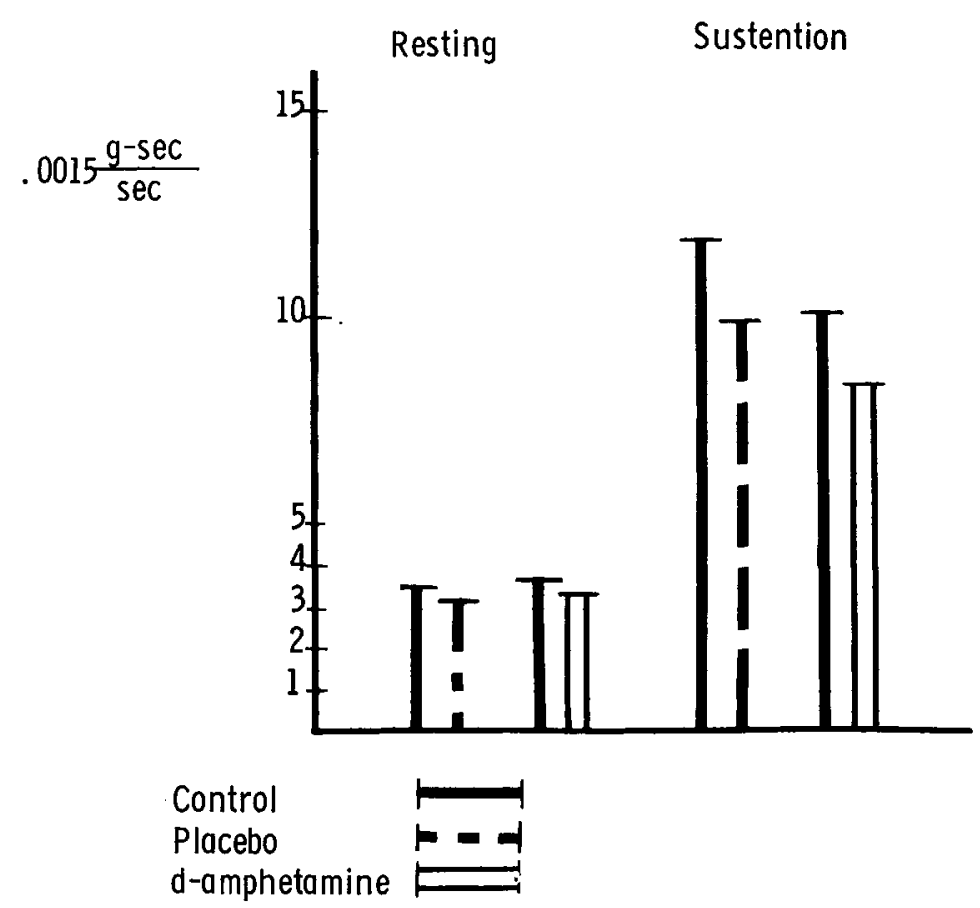

Fig. 1. Effects of $d$-amphetamine on resting and sustention tremor. The mean data are given for a predrug (control), placebo, and $10 \mathrm{mg}$. of $d$-amphetamine in this and the subsequent figures.

measure. For further description of the random and critical tracking tasks, see Repa and associates. ${ }^{8}$

The tests were conducted as follows: Subjects were asked to fast for 4 hours before the testing began. Then the complete test battery was conducted in the order described above, after which they were given $d$-amphetamine or placebo. The test battery took about one hour. After waiting 90 minutes, the subjects were retested. A urine specimen was taken and the subjective responses of the subjects were recorded. The subjects returned one week later and repeated the same procedure, this time receiving the alternate medication. Performance scores were obtained both before and after drug administration on each of the test days to account for the variability in performance resulting from testing on different days.

\section{Results}

Subjective impressions. Subjects were asked whether or not they could distin- guish any effects of the medications at the end of the placebo and $d$-amphetamine trials. Only 2 of the 6 subjects reported an effect following the $d$-amphetamine trial and no effect following the placebo trial. The others were unable to distinguish active drug from placebo.

Quantitative measures. The quantitative measures, comparing the control scores to the scores obtained after placebo and $d$-amphetamine administration are summarized in Table I. Paired $t$ tests were performed on the mean differences between placebo scores and $d$-amphetamine scores as well as on the mean differences between changes in scores following placebo and $d$-amphetamine administration. The results of these tests are also shown in Table I. The measures will be considered individually.

Tremor. The scores for the tremor tests shown in Table I are displayed in Fig. 1. For resting tremor, neither the differences between $d$-amphetamine scores and placebo nor the difference in the changes in scores following $d$-amphetamine and placebo ad- 


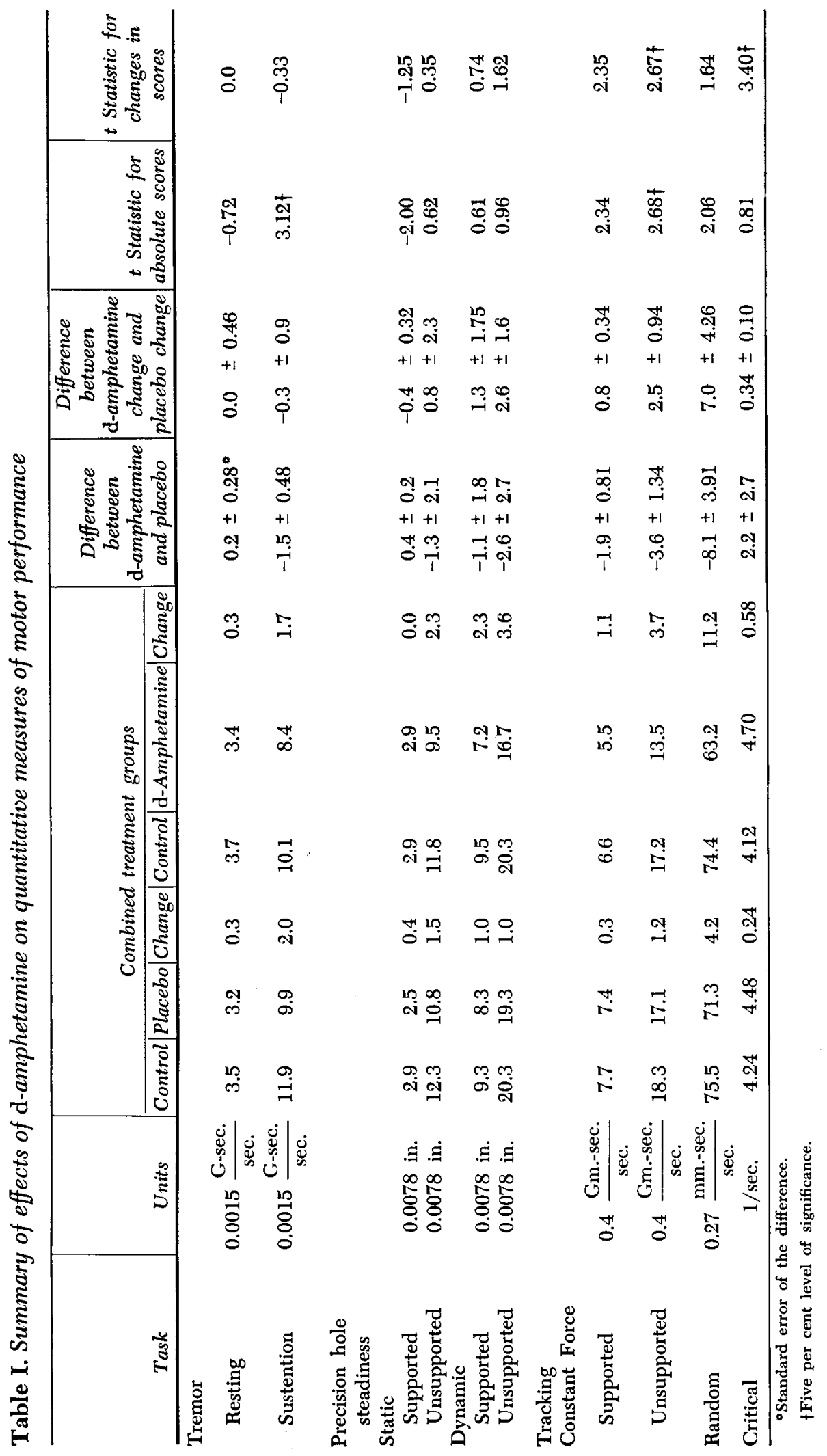


Static Supported Unsupported

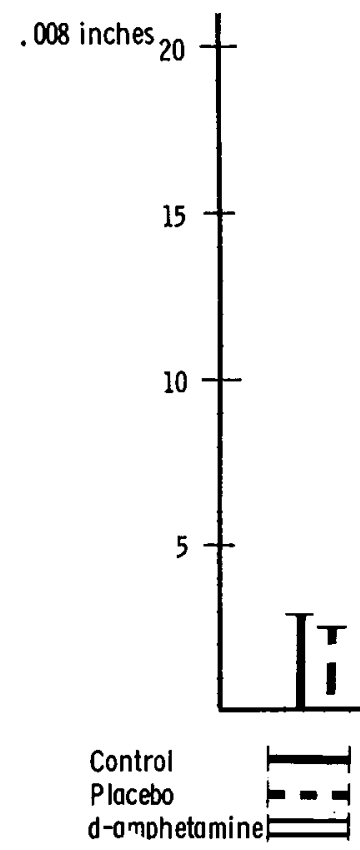

Dynamic

Supported Unsupported

Fig. 2. Effects of $d$-amphetamine on precision hole steadiness.

ministration was statistically significant. For sustention tremor there was a significant difference between $d$-amphetamine scores and placebo scores $(\mathrm{t}=3.12, \mathrm{p}<$ $0.05)$. However, examination of the sustention tremor control data indicates the large variability in this measure from one week to the next, suggesting that the change in sustention tremor following administration of placebo and $d$-amphetamine may be the more meaningful measure. When this measure was used there was no significant difference between $d$-amphetamine and placebo, although there was a slight trend for placebo to decrease sustention tremor to a greater extent than $d$-amphetamine.

Precision hole steadiness. The data for the precision hole steadiness tests are displayed graphically in Fig. 2 and numerically in Table I. There were no statistically significant differences between performance after placebo and after $d$-amphetamine for any of the measures, based either on absolute scores or changes in scores. In the static supported task, placebo performance was better than control performance, while $d$-amphetamine produced no change. This task required minimal motor output and is thought to be more sensitive to resting tremor levels than the other hole steadiness tests. In the three remaining steadiness tests, there was a tendency for both medications to improve performance, with $d$-amphetamine causing greater improvements in steadiness than placebo. These 3 tasks require more muscular output and coordination than the static supported task.

Tracking. The tracking scores are shown numerically in Table I and graphically in Fig. 3. The performance in the $d$-amphetamine trials tends to be better than that in the placebo trials for each of the tests based on both absolute scores and changes in scores. The change in performance with $d$ amphetamine was significantly greater than that with placebo for both the unsupported constant force task and the critical task ( $p$ $<0.05)$. There was also a significant difference in the absolute scores for $d$-am- 


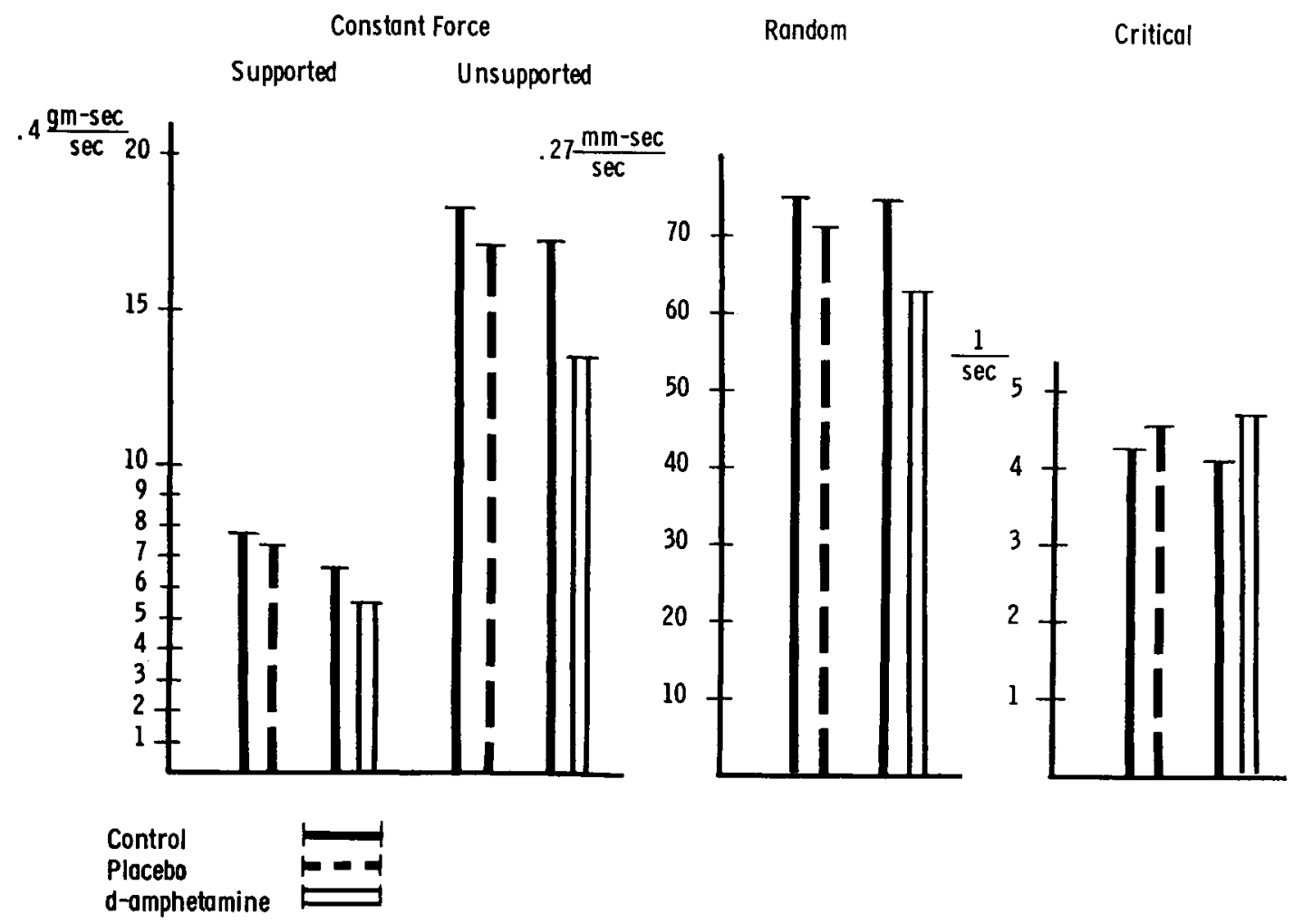

Fig. 3. Effects of $d$-amphetamine on tracking behavior under various conditions.

phetamine and placebo in the unsupported constant force task $(\mathrm{p}<0.05)$.

Urinary $p H$. At the end of $2 \frac{1}{2}$ hours after ingestion of either the placebo or $d$ amphetamine capsule, urinary $\mathrm{pH}$ was found to range from 6 to 7 for all subjects.

\section{Discussion}

A comparison has been made of absolute measures of tremor, precision hole steadiness, and tracking, as well as of changes in these measures following oral administration of placebo and $10 \mathrm{mg}$. of $d$-amphetamine in a double-blind, crossover trial. The data suggest that at the time of examination no significant changes in measures of tremor, resting and sustention, or precision hole steadiness can be attributed to $d$-amphetamine. However, several compensatory tracking tasks requiring sustained concentration and motor coordination did show significantly better performance after $d$ amphetamine than after placebo. Those tracking tasks that did not show statistical- ly significant differences, however, did show performance trends in favor of $d$ amphetamine. It appears that a $10 \mathrm{mg}$. dose of $d$-amphetamine does not significantly affect tremor or motor performance in tasks of short duration and minimal motor output. However, such a dose appears to significantly improve performance in complex motor tasks requiring more sustained subject attention and cooperation. The use of sensitive, quantitative testing procedures for evaluating small changes in motor performance is clearly justified. Of course, in evaluating over-all drug effects some measure of mood is also indicated.

These data are in line with previous observations in the literature. ${ }^{11}$ The advantage of some of the tasks described in this manuscript is their simplicity and especially their quantitative precision. Other compounds related to amphetamine would be of interest to compare.

It has been pointed out by Rowland and 
Beckett $^{9}$ that the oral absorption of $d$-amphetamine is complete about $1 \frac{1 / 2}{2}$ to $2 \frac{1}{2}$ hours after ingestion. It is for this reason that our subjects were studied between $1 \frac{1}{2}$ to $2 \frac{1 / 2}{2}$ hours after drug administration. It should also be pointed out that all of our subjects had a urinary $\mathrm{pH}$ between 6 and 7 at the end of the experimental session. Probably the variability in excretion due to changes in urinary $\mathrm{pH}$ therefore was decreased. Although the mechanisms of $d$-amphetamine action are very complex, ${ }^{2}$ the drug clearly produces behavioral effects in relatively small dosage in normal nonfatigued subjects.

\section{References}

1. Adler, H. F., Burckhardt, W. L., Ivy, A. C., and Atkinson, A. J.: Effect of various drugs on psychomotor performance at ground level and simulated altitudes of 18,000 feet in a low pressure chamber, Aviation Med. 21:221-236, 1950.

2. Costa, E., and Garattini, S.: International symposium on amphetamine related compounds, New York, 1970, Raven Press.

3. Eysenck, H. J., Casey, S., and Trouton, D. S.: Drugs and personality. IV. The effects of stimulant and depressant drugs on continuous work, J. Ment. Sci. 103:645-649, 1957.

4. Kornetsky, C., Mirsky, A. F., Kessler, E. K., and Dorff, J. E.: The effects of dextro-amphetamine on behavioral deficits produced by sleep loss in humans, J. Pharmacol. Exp. Ther. 127:46-54, 1959.

5. Kuzma, J. W., Tourtellotte, W. W., and Rem- ington, R. D.: Quantitative clinical neurological testing. II. Some statistical considerations of a battery of tests, J. Chron. Dis. 18: 303-311, 1965.

6. Payne, R. B., Hauty, G. T., and Moore, E. W.: Restoration of tracking proficiency as a function of amount and delay of analeptic medication, J. Comp. Physiol. Psychol. 50:145-149, 1957.

7. Potvin, A. R.: The effects of age, motivation, and learning on performance in the quantitative examination of neurological function. Doctoral dissertation, The University of Michigan, Ann Arbor, 1971.

8. Repa, B. S., Albers, J. W., Potvin, A. R., and Tourtellotte, W. W.: The use of a battery of tracking tests in the quantitative evaluation of neurological function. Seventh Annual NASAUniversity Conference on Manual Control, The University of Southern California, Los Angeles, California, June 2 to 4,1971 .

9. Rowland, M., and Beckett, A. H.: The amphetamines: Clinical and pharmacokinetic implications of recent studies of an assay procedure and urinary excretion in man, Arzneim. Forsch. 16:1369-1373, 1966.

10. Tourtellotte, W. W., Haerer, A. F., Simpson, J. F., Kuzma, J. W., and Sikorski, J.: Quantitative clinical neurological testing. I. A study of a battery of tests designed to evaluate in part the neurological functions of patients with multiple sclerosis and its use in a therapeutic trial, Ann. N. Y. Acad. Sci. 122:480-505, 1965.

11. Weiss, B., and Laties, V. G.: Enhancement of human performance by caffeine and the amphetamines, Pharmacol. Rev. 14:1-36, 1962.

12. Willett, R. A.: The effect of a stimulant and a depressant drug on the serial rote learning of nonsense syllables, Psychopharmacologia 3:23-34, 1962 . 